\title{
Positive schizotypy is associated with amplified mnemonic discrimination and attenuated generalization
}

Ágota Vass ${ }^{\mathrm{a}}$, Melinda Becske ${ }^{\mathrm{b}}$, Ágnes Szőllősi ${ }^{\mathrm{a}, \mathrm{c}}$, Mihály Racsmány ${ }^{\mathrm{a}, \mathrm{c}}$, Bertalan Polner ${ }^{\mathrm{a} *}$

${ }^{\text {a }}$ Department of Cognitive Science, Budapest University of Technology and Economics, Budapest, Hungary (postal address: 1111 Budapest, Hungary, Egry József u. 1.)

${ }^{\mathrm{b}}$ Department of Psychiatry and Psychotherapy, Semmelweis University, Budapest, Hungary (postal address: 1083 Budapest, Hungary, Balassa u. 6.)

${ }^{\mathrm{c}}$ Institute of Cognitive Neuroscience and Psychology, Research Centre for Natural Sciences, Budapest, Hungary (postal address: 1117 Budapest, Hungary, Magyar tudósok körútja 2.)

* Correspondence concerning this article should be addressed to Bertalan Polner at polner.bertalan@ttk.bme.hu. (Postal address: 1111-Budapest, Hungary, Egry József utca 1; telephone: +3614631273; fax: +3614631273 )

Author contributions: Bertalan Polner and Mihály Racsmány conceived and planned the experiments together with Ágota Vass, Melinda Becske, and Ágnes Szőllősi. Ágota Vass and Melinda Becske collected the data. Ágota Vass analysed the data, supervised by Bertalan Polner. Ágota Vass, Ágnes Szőllősi, Mihály Racsmány, and Bertalan Polner wrote the manuscript in consultation with Melinda Becske.

Acknowledgments: We are grateful to Anikó Szabados for contributing to data collection, to Ádám Markója for the technical assistance, and to Miklós Marián and Ádám Markója for supervising data collection. 
RUNNING HEAD: Schizotypy \& pattern separation/false memory

\begin{abstract}
Tendency to experience inaccurate beliefs alongside perceptual anomalies constitutes positive schizotypal traits in the general population and shows continuity with the positive symptoms of schizophrenia. It has been hypothesized that the positive symptomatology of schizophrenia, and by extension, the odd beliefs and unusual perceptual experiences in the general population, are associated with specific alterations in memory functions. Imbalance between memory generalization and episodic memory specificity has been proposed on several counts; however, the direction of the imbalance is currently unclear. Here, we evaluated the association between positive schizotypy, and memory alterations related to hippocampal computations in a general population sample enriched for positive schizotypy. We found that memory generalization is attenuated while memory specificity is elevated in participants with more pronounced positive schizotypal traits. Our findings show that people who are prone to irrational beliefs and unusual experiences also show measurable alterations in memory and likely have difficulty grasping the global picture and rather be overpowered by fragments of information.
\end{abstract}

Keywords: hippocampus; memory; odd beliefs; pattern completion; pattern separation 
RUNNING HEAD: Schizotypy \& pattern separation/false memory

\section{Introduction}

Schizotypal personality traits have been conceptualized to form a continuum with symptoms of schizophrenia [1] which is evidenced by marked phenomenological and cognitive overlaps $[1,2]$ and higher transition probability of people with more pronounced schizotypal traits into psychosis [3]. While schizophrenia is a severe neuropsychiatric disorder with a prevalence rate of under 1\% [4], schizotypal traits represent a mild analogue of the symptoms of schizophrenia and form part of the natural variation of personality traits in the general population $[1,3,5]$. Schizotypy comprises multiple dimensions, one of which is positive schizotypy, that implies odd, delusion-like beliefs, and unusual, hallucination-like experiences [1].

It has been suggested that the positive symptoms of schizophrenia are related to specific learning and memory alterations [6] implying that people who are prone to irrational beliefs and unusual perceptions may also demonstrate distinct alterations in memory functions. There is a discrepancy in the literature, however, regarding how positive symptoms are related to such alterations in memory. According to Tamminga and colleagues, the positive symptomatology of schizophrenia is linked to the imbalance of hippocampal neural computations $[7,8]$. The hippocampus is a medial temporal lobe structure that is associated with episodic memory [9] and is assumed to play a leading role in memory specificity and generalization [10]. Memories are likely to overlap with each other in content which hinders the retrieval of unique memories. Storing specific details in the face of significant overlap between memory representations is carried out by pattern separation, a neural computation in the hippocampus. As a result of pattern separation, the neuronal activities of brain circuits become distinct for two or more stimuli that share similar features $[11,12]$. On the other hand, the unification of related memory elements into an integrated unit is attributed to the neural computation of pattern completion. Pattern completion relies on an autoassociative network in the hippocampus that fills in incomplete incoming information based on previously stored representations [11]. It is, thus, theorized to play a role in generalization $[10,12]$.

While it is assumed that the imbalance between neural computations related to episodic memory is associated with positive symptoms, the direction of the imbalance is unclear. On the one hand, increased memory generalization and deteriorated specificity were assumed to share a common ground 
RUNNING HEAD: Schizotypy \& pattern separation/false memory

with hallucinations and delusions $[7,8]$. In terms of underlying neural mechanisms, this translates into amplified pattern completion and attenuated pattern separation, as pattern completion is assumed to be associated with memory generalization and pattern separation is assumed to be related to memory specificity [10]. The findings of Das and colleagues [13], Martinelli and Shergill [14], as well as Kraguljac and colleagues [15] supported this framework of impaired memory specificity in schizophrenia. All studies found the behavioural manifestation of pattern separation to be weaker in schizophrenia, however, no relationship between the positive symptoms and memory deficits was revealed. The results are likely to be confounded by relatively small samples, the effect of antipsychotic medications [16] and may reflect a generalized performance deficit rather than a function-specific alteration [17].

While these studies highlight that impaired memory specificity may be related to the positive symptoms of schizophrenia, the long-standing literature of the disorder that dates as far back as to the 1900's suggests otherwise [18]. Schizophrenia has been described as a disorder of fragmentation with a distorted sense of subjectivity [19] and by the loss of sense of continuity both to one's self and perceptual organization [20]. Relatedly, studies specifically assessing memory alterations have found that positive symptoms correlated with an increased tendency to remember the local as opposed to the global features of the stimuli $[21,22]$. Such alterations are rather translatable into amplified memory specificity, implying overactive pattern separation and impaired generalization, which would correspond to attenuated pattern completion at the neural level.

\subsection{Motivation and Aims}

It is currently unclear how memory formation may be altered in relation to the positive symptoms of schizophrenia and, by extension, in people who are prone to irrational or delusion-like belief formation and unusual perceptual experiences. In particular, two opposing hypotheses emerged from the literature. On the one hand, the work of Tamminga and colleagues $[7,8]$, as well as empirical studies examining clinical samples [13-15] supported the hypothesis that memory specificity is deteriorated, and generalization is enhanced in schizophrenia. On the other hand, countless accounts in the long-standing literature on memory alterations in schizophrenia [21-24] and general phenomenology 
RUNNING HEAD: Schizotypy \& pattern separation/false memory

of the disorder $[18,19]$ argue that it is rather the process of generalization that is defective, and the disorder leaves people incapacitated to integrate fragments into a whole implying amplified specificity.

Here, for the first time, we aimed to contrast these two competing hypotheses. To avoid confounding by illness-related factors such as hospitalization and medication, we recruited a sample from the general population. To achieve higher statistical power, we increased variability in the sample by oversampling for positive schizotypy. We assessed memory specificity and generalization - as behavioural indicators of pattern separation and completion, respectively - with a modified recognition memory task, the well-established Mnemonic Similarity Task [MST; 25]. We fitted linear regression models to evaluate the relationship between positive schizotypy and memory specificity vs. generalization. We established the high reliability of our key measurements. Robustness of the associations were tested by control analyses adjusting the models for age and gender, perceptual deficits, negative, disorganized, and impulsive dimensions of schizotypy, and additional aspects of psychopathology.

\section{Materials and Methods}

\subsection{Participants}

We have oversampled for high positive schizotypy to ensure sufficient variability, as our aim was to specifically focus on the positive dimension. As part of the oversampling, 614 people filled out an online form that included the positive and negative schizotypy subscales from the short OxfordLiverpool Inventory of Feelings and Experiences Questionnaire (O-LIFE; for details, see below) and a question about the age of the respondent. The form selected participants based on the following criteria: age between 18 and 35 years, achieve 7 out of 12 points or higher on the Unusual Experiences subscale that assesses positive schizotypy, and 3 out of 12 points or lower on the Introvertive Anhedonia subscale that assesses negative schizotypy (these cut-offs were based on previous large university student samples [e.g. 26, 27].

191 people met the criteria and 23 of them volunteered to participate in the study. In addition, a convenience sample of 64 participants were recruited through university courses and social media advertisements. Some of the participants received course credit as a form of compensation. Further, 11 out of the total of 87 participants were excluded from the final analysis due to a self-reported history of 
RUNNING HEAD: Schizotypy \& pattern separation/false memory

neurological or psychiatric disorders, epilepsy, or traumatic brain injury. Five participants were excluded because they were identified by Cook's Distance as influential points distorting the results of our regression models (see details in Results). Thus, the final sample comprised 71 participants (17 males, mean [SD] age: 24.0 [5.1] years, mean [SD] education: 16.1 [2.3] years).

Participants provided written informed consent. The authors assert that all procedures contributing to this work comply with the ethical standards of the relevant national and institutional committees on human experimentation and with the Helsinki Declaration of 1975, as revised in 2008. The study was approved by the United Ethical Review Committee for Research in Psychology, Hungary $(2016 / 032)$.

\subsection{Questionnaires}

Participants completed several self-administered psychometric instruments to quantify their level of schizotypy and general mental well-being from a variety of perspectives. Overall, the instruments had good reliability in the sample $(\alpha>=0.7$, except for negative and impulsive schizotypy; see details in Supplementary Materials and distributions in Supplementary Figure 1). Participants completed the short version of the O-LIFE questionnaire [28] [Hungarian version: , 29] which measures schizotypal personality traits. As sleep disturbances have been shown to be correlated with schizotypy [30], participants completed the Athen Insomnia Scale (AIS) [31] [Hungarian version: , 32]. To obtain information on psychopathological states, participants also filled questionnaires about momentary psychotic-like experiences [33] and state anxiety [34] [Hungarian version: , 35]. Higher scores in all questionnaires correspond to an indicator of pathological functioning, except for the General Health Questionnaire-12 (GHQ-12) [36] [Hungarian version: , 37], where a higher score corresponds to better mental health.

\subsection{Mnemonic Similarity Task}

The task is illustrated in Figure 1. In the incidental encoding phase of the task, participants made simple decisions about pictures of everyday objects. Unbeknownst to participants, this was followed by a recognition phase, where participants were asked to make memory judgements about pictures of everyday objects that were either identical, visually similar, or completely different from the pictures shown in the encoding phase (targets, lures, and foils, respectively). Following previous studies [see 
RUNNING HEAD: Schizotypy \& pattern separation/false memory

25], the discrimination performance between the studied old items and their visually similar lure pictures (the ratio of "similar" responses given to the lure items) was used as an indicator of memory specificity (widely known as the so-called Lure Discrimination Index; LDI), while false recognition of similar lure pictures (the ratio of "old" responses given to the lures) was used as an index of memory generalization. Both indices had good split-half reliability in the sample ( 0.80 and 0.78 , respectively). For the detailed description of the task, see Supplementary Materials and [25].

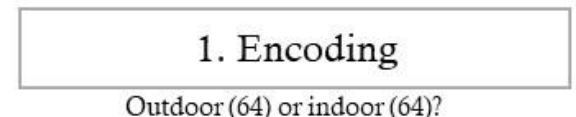

Outdoor (64) or indoor (64)?

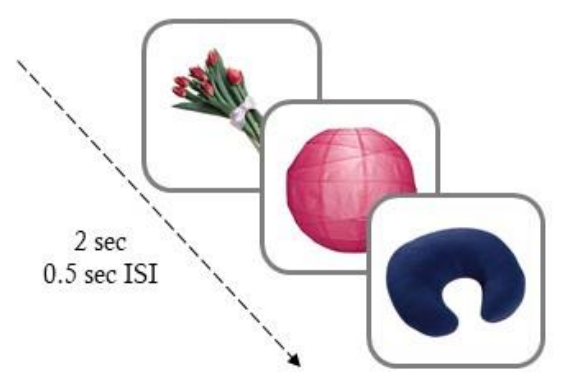

2. Recognition

Foil (64), lure (64) or target (64)?

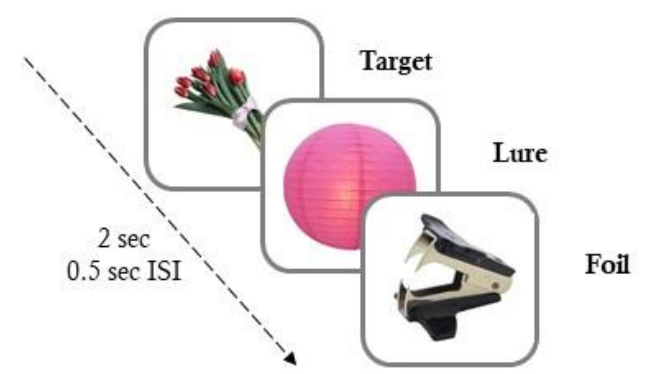

Fig. 1 The design and the experimental procedure of the Mnemonic Similarity Task.

Note(s). Participants were presented 128 pictures of everyday objects in the encoding phase. In the recognition phase, participants were shown 192 pictures of everyday objects, 64 of which were exact repetitions of objects shown in the encoding phase (targets), 64 were new objects (foils) and 64 were perceptually similar items to ones presented in the encoding phase of the task (lures). ISI = inter-stimulus interval

\subsection{Perceptual Discrimination Task}

To control for perceptual deficits, participants took part in a Perceptual Discrimination (PD) test immediately after the completion of the MST. Participants judged whether 90 pairs of images were identical to, different from, or similar to each other. Stimuli were colourful images of objects. Each trial consisted of the subsequent presentations of two images and participants were asked to identify them 
RUNNING HEAD: Schizotypy \& pattern separation/false memory

correctly as "old", "new", or "similar" via button press. For the detailed description of the task, see Supplementary Materials.

\section{Results}

We have carried out a series of linear regression analyses to examine the relationship between behavioural indicators of pattern separation (lure discrimination index; LDI) and pattern completion (false recognition of the lure stimuli) and positive schizotypy scores. The assumptions of homoscedasticity and normally distributed residuals had been tested and met. Since data points with large residuals or high leverage may distort the outcome and accuracy of a regression model, we have excluded five participants based on the Cook's distance. To assess the specificity of the relationships between positive schizotypy and the LDI as well as the false recognition of lures, we have carried out control analyses, where the model was extended with age and gender (entered simultaneously) or one of the following variables: perceptual discrimination performance; negative, disorganised, or impulsive schizotypy; insomnia; general mental health; momentary psychotic-like experiences; state anxiety. The results are summarised in Table 1.

\subsection{Lure Discrimination/False Recognition of Lures and Positive Schizotypy}

Firstly, we investigated the relationship between the LDI as well as false recognition of lures and positive schizotypy. If a higher score on the unusual experiences scale of the O-LIFE questionnaire is to be associated with an attenuated LDI and a higher number of false recognition of lures, then our results would lend support to the hypothesis of impaired memory specificity and enhanced generalization. However, if higher positive schizotypy scores are to be associated with an amplified LDI and lower false recognition of lures, then our results would be in line with the hypothesis of impaired memory generalization and enhanced specificity. Critically, we found that positive schizotypy was significantly related to enhanced lure discrimination and lower false recognition of lures (see Table 1, Positive schizotypy section and Figure 2A and 2C). In the following analyses, we evaluated the robustness and specificity of these associations. 
RUNNING HEAD: Schizotypy \& pattern separation/false memory

Table 1. Summary of linear regression models predicting lure discrimination/false recognition of lures from positive schizotypy, before and after adjustment for control variables.

\begin{tabular}{|c|c|c|c|c|c|c|}
\hline \multirow[b]{2}{*}{ Predictor } & \multicolumn{3}{|c|}{ LDI } & \multicolumn{3}{|c|}{ False recognition of lures } \\
\hline & $\beta[95 \% \mathrm{CI}]$ & $F$ & $R^{2}$ & $\beta[95 \% \mathrm{CI}]$ & $F$ & $R^{2}$ \\
\hline \multicolumn{7}{|c|}{ Positive schizotypy } \\
\hline Positive schizotypy & $\begin{array}{l}0.017^{* * *} \\
{[0.0048} \\
0.0297]\end{array}$ & $7.67^{* * * *}$ & 0.1 & $\begin{array}{l}-0.018^{* * *} \\
{[-0.0280} \\
-0.0087]\end{array}$ & $14.33^{* * *}$ & 0.17 \\
\hline \multicolumn{7}{|c|}{ Age and gender } \\
\hline Positive schizotypy & $\begin{array}{l}0.015^{* *} \\
{[0.0029} \\
0.0279]\end{array}$ & $3.57^{* *}$ & 0.14 & $\begin{array}{l}-0.016^{* * *} \\
{[-0.0259} \\
-0.0068]\end{array}$ & $7.07^{* * *}$ & 0.24 \\
\hline Age & $\begin{array}{l}-0.006^{*} \\
{[-0.0134} \\
0.0010]\end{array}$ & & & $\begin{array}{l}0.007^{* *} \\
{[0.0012} \\
0.0122]\end{array}$ & & \\
\hline Gender & $\begin{array}{l}-0.010 \\
{[-0.0945} \\
0.0752]\end{array}$ & & & $\begin{array}{l}0.020 \\
{[-0.0447} \\
0.0846]\end{array}$ & & \\
\hline
\end{tabular}

\begin{tabular}{|c|c|c|c|c|c|c|}
\hline \multicolumn{7}{|c|}{ Perceptual discrimination (PD) } \\
\hline Positive schizotypy & $\begin{array}{l}0.016^{* *} \\
{[0.0031} \\
0.0291]\end{array}$ & $3.98^{* *}$ & 0.1 & $\begin{array}{l}-0.018^{* * *} \\
{[-0.0286} \\
-0.0078]\end{array}$ & $7.07^{* * * *}$ & 0.17 \\
\hline $\begin{array}{l}\mathrm{LDI} / \text { False recognition of lures on } \\
\text { the PD task }\end{array}$ & $\begin{array}{l}0.082 \\
{[-0.1885} \\
0.3526]\end{array}$ & & & $\begin{array}{l}0.022 \\
{[-0.5071} \\
0.5514]\end{array}$ & & \\
\hline
\end{tabular}

\begin{tabular}{|c|c|c|c|c|c|c|}
\hline \multicolumn{7}{|c|}{ Schizotypal traits } \\
\hline Positive schizotypy & $\begin{array}{l}0.016^{* *} \\
{[0.0034} \\
0.0291]\end{array}$ & $4.03^{* *}$ & 0.11 & $\begin{array}{l}-0.016^{* * *} \\
{[-0.0263} \\
-0.0066]\end{array}$ & $8.77^{* *}$ & 0.21 \\
\hline Negative schizotypy & $\begin{array}{l}-0.010 \\
{[-0.0379} \\
0.0187]\end{array}$ & & & $\begin{array}{l}0.018^{*} \\
{[-0.0034} \\
0.0400]\end{array}$ & & \\
\hline Positive schizotypy & $\begin{array}{l}0.017^{* *} \\
{[0.0031} \\
0.0300]\end{array}$ & $3.82^{* *}$ & 0.1 & $\begin{array}{l}-0.020^{* * *} \\
{[-0.0306} \\
-0.0098]\end{array}$ & $7.61^{* * * *}$ & 0.18 \\
\hline Disorganized schizotypy & $\begin{array}{l}0.002 \\
{[-0.0131,} \\
0.0172]\end{array}$ & & & $\begin{array}{l}0.006 \\
{[-0.0062} \\
0.0173]\end{array}$ & & \\
\hline Positive schizotypy & $\begin{array}{l}0.015^{* *} \\
{[0.0007} \\
0.0293]\end{array}$ & $4.00^{* * *}$ & 0.11 & $\begin{array}{l}-0.015^{* * *} \\
{[-0.0265} \\
-0.0044]\end{array}$ & $7.78^{* * *}$ & 0.19 \\
\hline
\end{tabular}


RUNNING HEAD: Schizotypy \& pattern separation/false memory

\begin{tabular}{l|l|l} 
Impulsive schizotypy & 0.007 & -0.010 \\
{$[-0.0159$,} & {$[-0.0279$,} \\
& $0.0307]$ & $0.0082]$ \\
\hline
\end{tabular}

Further trait- and state-like psychopathology

\begin{tabular}{|c|c|c|c|c|c|c|}
\hline $\begin{array}{l}\text { Positive schizotypy } \\
\text { Insomnia }\end{array}$ & $\begin{array}{l}0.017^{\text {**** }} \\
{[0.0043,} \\
0.0294] \\
0.003 \\
{[-0.0082,} \\
0.0141]\end{array}$ & $3.93^{* *}$ & 0.1 & $\begin{array}{l}-0.018^{* * *} \\
{[-0.0278} \\
-0.0082] \\
-0.003 \\
{[-0.0115} \\
0.0059]\end{array}$ & $7.31^{* * *}$ & 0.18 \\
\hline $\begin{array}{l}\text { Positive schizotypy } \\
\text { General mental health }\end{array}$ & $\begin{array}{l}0.017^{* * * *} \\
{[0.0046,} \\
0.0297] \\
-0.001 \\
{[-0.0090,} \\
0.0065]\end{array}$ & $3.83^{* *}$ & 0.1 & $\begin{array}{l}-0.018^{* * *} \\
{[-0.0280,} \\
-0.0085] \\
0.001 \\
{[-0.0047} \\
0.0073]\end{array}$ & $7.17^{* * * *}$ & 0.17 \\
\hline $\begin{array}{l}\text { Positive schizotypy } \\
\text { Psychotic experiences }\end{array}$ & $\begin{array}{l}0.021^{\text {**** }} \\
{[0.0074,} \\
0.0340] \\
-0.005 \\
{[-0.0116,} \\
0.0021]\end{array}$ & $4.86^{* *}$ & 0.13 & $\begin{array}{l}-0.021^{* * *} \\
{[-0.0313,} \\
-0.0106] \\
0.004 \\
{[-0.0018} \\
0.0089]\end{array}$ & $8.14^{* * *}$ & 0.19 \\
\hline Positive schizotypy & $\begin{array}{l}0.017^{* * *} \\
{[0.0049,} \\
0.0298] \\
-0.002 \\
{[-0.0060,} \\
0.0028]\end{array}$ & $4.07^{* *}$ & 0.11 & $\begin{array}{l}-0.018^{* * *} \\
{[-0.0282,} \\
-0.0087] \\
0.001 \\
{[-0.0025} \\
0.0044]\end{array}$ & $7.26^{* * * *}$ & 0.18 \\
\hline
\end{tabular}

$\overline{\text { Note }(s) \text {. LDI: Lure Discrimination Index. When adjusting for performance on the perceptual discrimination (PD) }}$

task, the LDI on the PD was added when predicting the LDI on the MST, and false recognition of lures on the PD task was added when predicting false recognition of lures on the MST. For the $F$-test, degrees of freedom are 2 and 68, except for the first model where positive schizotypy is the only predictor, where degrees of freedom are 1 and 69, and the model with the demographic control variables, where degrees of freedom are 3 and $67 . N=71$, * $p<.05,{ }^{* *} p<.01,{ }^{* * * *} p<.001$

\subsection{Control Analyses: Age and Gender}

We have examined whether the significant relationship between positive schizotypy and the LDI and false recognition of lures prevails when age and gender are adjusted for. The rationale for conducting this control analysis was that discrimination performance is known to be affected in the 
RUNNING HEAD: Schizotypy \& pattern separation/false memory

elderly [e.g. 25, 38]. Based on the results, the relationship with positive schizotypy was still robust (see Table 1, Age and gender section, and Figures 3D and 3H). However, we should also note that age showed a significant relationship with the hippocampal computations that are of interest to us. Attenuated mnemonic discrimination performance and enhanced false recognition of lures were more likely to be observed in relatively older participants in our sample.

\subsection{Control Analyses: Perceptual Discrimination}

Further, we considered the possibility that deficits in perception and early visual information processing may cascade to later stages of information processing. Thus, we have examined whether the significant effects detected could be explained by elementary perceptual deficits [14]. For this purpose, we have estimated the LDI and false recognition of lures on the PD test for each individual. Our results showed a significant relationship between performance on the PD test and the O-LIFE Unusual Experiences score (see Figure 2B and 2D). Higher positive schizotypy was associated with enhanced lure discrimination performance $(\beta(\mathrm{SE})=0.014(0.006), p<0.01, F(1,69)=5.997, p<0.01$, Adjusted $\left.R^{2}=0.067\right)$ and less false recognitions of lures $(\beta(\mathrm{SE})=-0.007(0.002), p<0.001, F(1,69)=9.785, p<$ 0.001 , Adjusted $R^{2}=0.112$ ) on the PD test. However, discrimination and false recognition of lures on the MST were not significantly associated with PD test performance, and the effect of positive schizotypy on performance on the MST remained significant after adjusting for the non-significant effects of PD indices (see Table 1, Perceptual discrimination section). Overall, these findings suggest that, although positive schizotypy is associated with altered perceptual discrimination performance, this does not explain the observed alterations in memory specificity and generalization. 
RUNNING HEAD: Schizotypy \& pattern separation/false memory
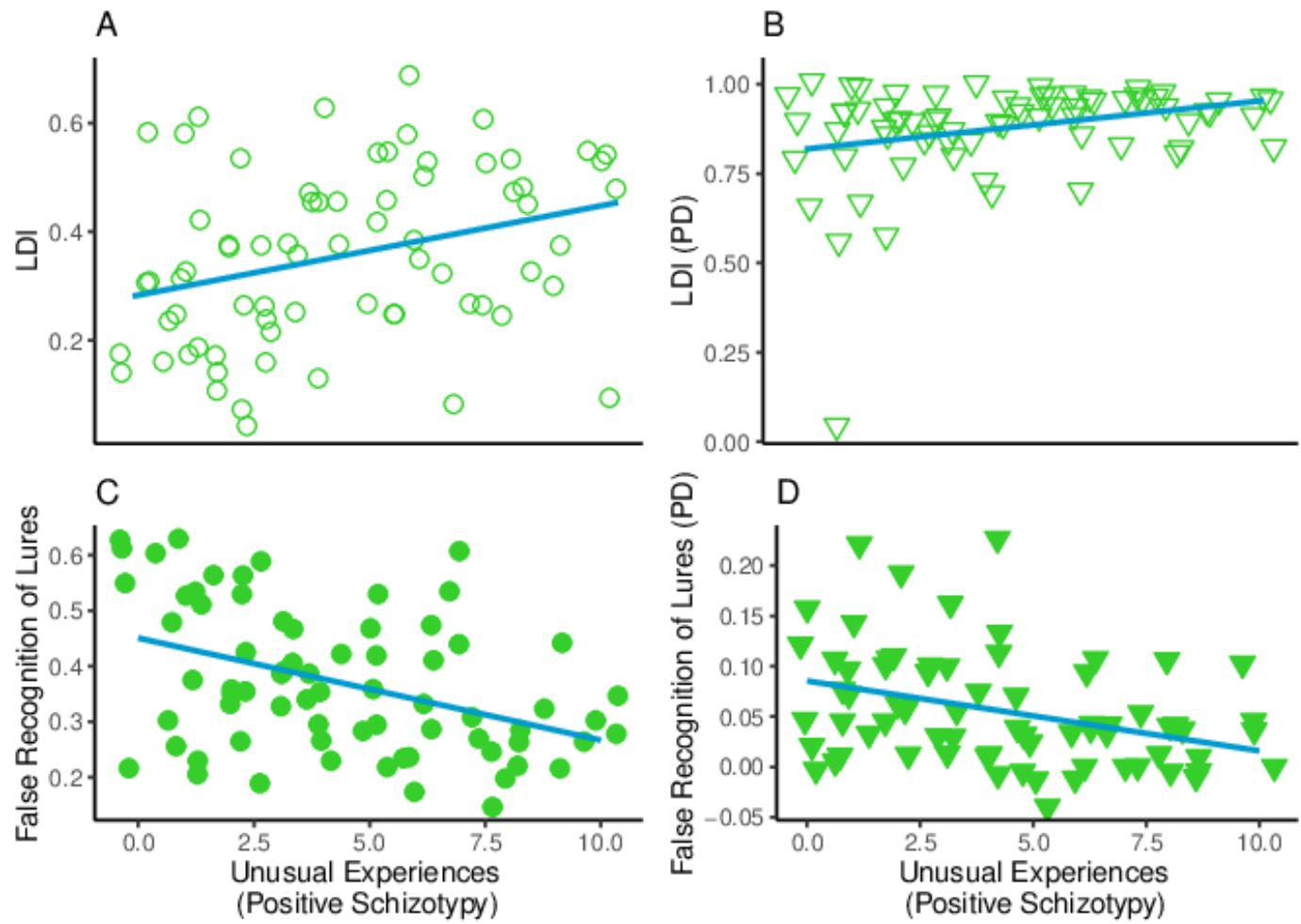

Fig. 2 The association between positive schizotypy (O-LIFE) and lure discrimination/false recognition of lures achieved in the MST and PD tests.

Note(s). The $x$-axis shows positive schizotypy and the $y$-axis shows the MST ('A' and 'C') and PD performance scores ('B' and 'D'). Linear trendlines with 95\% CI are shown

\subsection{Control Analyses: Negative, Disorganized and Impulsive-Asocial Dimensions of Schizotypy}

We considered three additional dimensions of schizotypy beyond positive schizotypy and examined whether the relationship between putative behavioural indicators of hippocampal neural computations and positive schizotypy is specific, or whether it can be extended to the negative, disorganized, and impulsive-asocial dimensions of schizotypy. None of these O-LIFE dimensions had a statistically significant relationship with LDI when added as second explanatory variables, and only negative schizotypy had a statistically significant and positive effect on the false recognition of lures (see Table 1, Schizotypal traits section and Figure 3A-C and Figure 3E-G). After adjusting for this effect, the associations between positive schizotypy and lure discrimination as well as the false recognition of lures did not change essentially, providing evidence for their robustness. 


\subsection{Control Analyses: Further Trait- and State-Like Psychopathology}

To further test for the robustness of the effect of positive schizotypy, we have constructed regression models where we added insomnia, general mental health, momentary psychotic-like experiences, and state anxiety, respectively, as additional explanatory variables alongside positive schizotypy. Our results showed that none of them had a statistically significant effect on the LDI or the false recognition of lures, and the effect of positive schizotypy was robust (see Table 1, Further traitand state-like psychopathology section and Figure 3I-P).
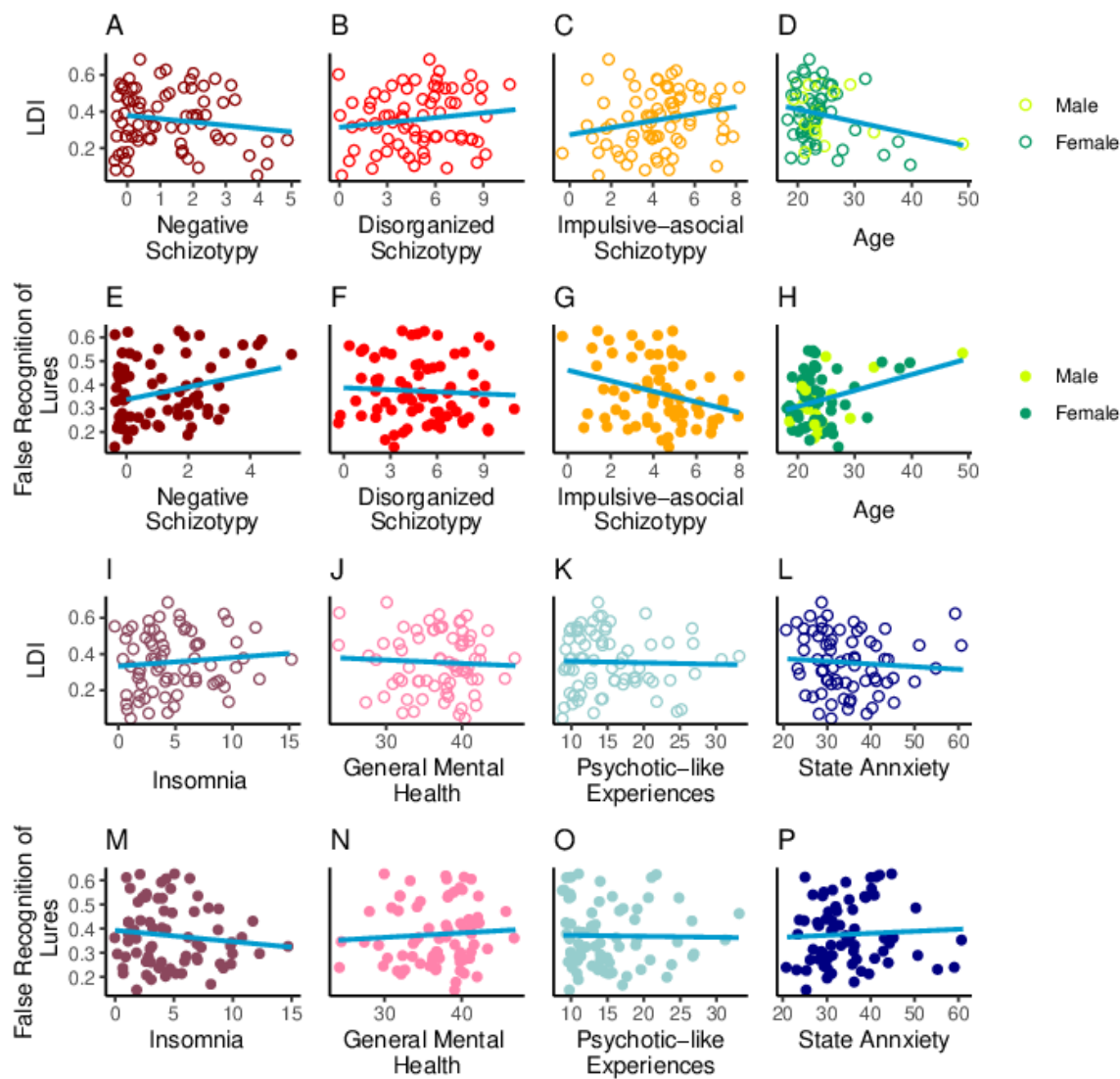

Fig. 3 The association between negative, disorganized and impulsive-asocial dimensions of schizotypy, age and gender, further trait- and state-like psychopathology and lure discrimination/false recognition of lures achieved in the MST.

Note(s). The $x$-axis shows the different schizotypy dimensions ('A'- 'C', 'E'-'G'), age and gender ('D', 'H'), and further trait- and state-like psychopathology ('I'-'P'). The $y$-axis shows the MST performance scores. Linear trendlines with $95 \% \mathrm{CI}$ are shown 
RUNNING HEAD: Schizotypy \& pattern separation/false memory

\section{Discussion}

Positive schizotypy in the general population refers to a tendency of experiencing perceptual aberrations and odd beliefs, and it seems to form a continuum with the positive symptoms of schizophrenia such as hallucinations and delusions [1]. It has been established that these symptoms are associated with specific memory alterations, however, two opposing hypotheses exist that outline the nature of these alterations. Our aims in the current study were twofold. Firstly, as there is a considerable phenomenological and cognitive overlap between schizophrenia and schizotypy [1], we tested whether people with elevated positive schizotypal traits also demonstrate specific alterations in memory. Importantly, by recruiting individuals from the general population, we minimized confounding by illness-related factors such as medication or hospitalization, which was a potential limitation of previous patient studies. Secondly, we aimed to evaluate the two contrasting hypotheses and test whether people with more pronounced positive schizotypal traits were more prone to overgeneralise when remembering or memorising rather the local than the global features of events.

Specifically, in line with previous work studying memory generalization and specificity at the behavioural level, we adapted the MST [25] to measure the behavioural manifestation of specific hippocampal neural computations. The LDI corresponded to the behavioural proxy of pattern separation and provided an estimate of the ability to distinguish perceptually similar stimuli, thus, to discriminate between overlapping memory representations. False recognition of lures corresponded to the behavioural proxy of pattern completion and served as an estimate of the capability to generalize across perceptually similar features. Over and above the effects of age and gender, perceptual discrimination, other dimensions of schizotypy and associated psychopathology, positive schizotypy was consistently associated with enhanced memory specificity and attenuated generalization. Thus, our results support the hypothesis that has arisen from the long-established literature describing schizophrenia as a disorder of fragmentation at several levels $[18,39]$.

\subsection{Impaired Generalization}

We found that the more pronounced positive schizotypal traits an individual possesses, the lower their ability is to mnemonically generalize. This is in line with evidence suggesting that generalization processes are compromised in schizophrenia [16, 40]. It has been argued that generalization and pattern 
RUNNING HEAD: Schizotypy \& pattern separation/false memory

completion are related concepts [10]. Relatedly, attenuated pattern completion can be conceptualized as one possible mechanism underlying lower generalization performance.

According to influential models of autobiographical memory, episodic memories are formed by a balanced interaction of encoded experience-near record of ongoing activity and the simultaneous need to maintain a coherent and stable record of the self's interaction with the world that extends beyond the present moment $[41,42]$. Extracting commonalities regarding one's self from different experiences which are robust and permanent presumably requires the ability to generalize. Relatedly, it has been argued that a cohesive self develops out of an interaction between numerous 'selves' that are experienced through a wide variety of different situations [43]. In line with the theory of weaker generalization processes, people diagnosed with schizophrenia were also found to have a decreased selfconcept clarity [43]. This implies that patients show impairment in the generalization of common features regarding the self from various events. Specifically, self-concept clarity was negatively associated with positive symptoms of schizophrenia. Additionally, attenuated self-concept clarity has also been found to co-occur with the positive, negative, and disorganized factors of schizotypy $[44,45]$.

Taken together, impairments in the ability to generalize across several domains in schizophrenia and schizotypy strongly support our findings that the behavioural proxy of pattern completion is negatively linked to the positive dimension of schizotypy, implying that higher positive schizotypal traits are associated with a compromised ability to generalize.

\subsection{Amplified Specificity}

Higher positive schizotypy was associated with enhanced memory specificity in the present study. This finding is in line not only with previous findings, but it may also highlight a core, unifying and more central feature of schizophrenia. Bleuler [18] conceptualized schizophrenia as "a mind that is torn asunder" by loosening associations [cited by 46]. He suggested that the fragmentation of thought, emotion and volition should be viewed as the core psychological feature of the disorder [39]. While the inherently subjective nature of fragmentation renders it challenging to measure, it manifests itself in pervasive disturbances in mental processes, some of which are observable and quantifiable. Evidence suggests that individuals with schizophrenia often struggle with multisensory integration $[47,48]$. More importantly, difficulties in information integration within a single cognitive domain such as memory 
RUNNING HEAD: Schizotypy \& pattern separation/false memory

have also been reported. During visual memory tests, patients exhibited a detail-oriented style of processing and impaired ability to integrate the details into global features [23, 24]. These results correspond to the theory that there is a local, as opposed to global, processing bias in schizophrenia [22]. Further, Ferman et al [21] used the global-local paradigm in relation to the different dimensions of the disorder. Importantly, they found that in patients exhibiting positive symptoms, there was a propensity towards local processing which, in turn, interfered with global processing. They argued that this provided evidence for a heightened distractibility to feature detail in association with positive symptoms of schizophrenia [21].

Our finding of amplified specificity in high positive schizotypy contrasts with previous studies in patients with schizophrenia which reported impaired memory specificity [13-15]. We suggest that this discrepancy might be resolved by considering the heterogeneity of schizophrenia: impaired memory specificity might be a marker of accelerated brain ageing in schizophrenia $[49,50]$. This would be in line with the well-established finding of a positive correlation between impaired memory specificity/lure discrimination/pattern separation and age [38, 51-53] [12, for overviews, see e.g. , 25]. On the other hand, the positive symptom dimension might be related to increased memory specificity.

Overall, disruption in the integration of information and, in particular, shift towards detailoriented processing is evident from studies. Our results support some of the earliest phenomenological descriptions of schizophrenia in the literature [18] and are also in line with studies investigating memory alterations in the disorder in that we have found memory specificity to be enhanced in people with higher positive schizotypy.

\subsection{Strengths and limitations}

The strengths of the study include the comparison of two well-articulated competing hypotheses, the evaluation of alternative explanations through a series of control analyses, and the reliable measurement of psychopathology and memory alterations. One may argue that our study is limited by the sample size. However, it should be noted that in order to achieve higher statistical power, we increased variability in the sample by oversampling for positive schizotypy, and we also established the high reliability of our key measurements. 
RUNNING HEAD: Schizotypy \& pattern separation/false memory

\section{Conclusion}

Deficits in memory, perception, attention, language production, or motor control have all been detected in schizophrenia and, with lower effect sizes, in people showing high levels of schizotypal traits $[1,2]$. Memory dysfunction is considered to be a particularly pronounced symptom of schizophrenia [54], as well as being one of the strongest predictors of illness outcome [55, 56], however, opposing hypotheses emerged in the literature regarding whether patients are more biased toward overgeneralization, or detail-oriented memories. Over and above contrasting the two frameworks, our aim was to investigate whether people with higher positive schizotypal traits in the general population would also show a specific bias in memory. Our results suggest that people who are prone to odd, delusion-like beliefs, and unusual, hallucination-like experiences are also more likely to struggle to get hold of the global picture. The fragmentation of memory representations has an important implication on how we interpret the world around ourselves and how we synthesize information. Thus, people with higher positive schizotypal traits may be more susceptible to experience unusual perceptions and come to erroneous conclusions as they may be unable to look beyond the details and take the broad picture into account. Future high-powered studies with patients should examine the relationship between variation in symptoms and behavioural indicators of pattern separation and completion.

\section{Declarations}

\section{Funding}

BP was supported by the BME-Biotechnology FIKP grant of EMMI (BME FIKP-BIO), and by the National Research, Development and Innovation Office (NKFI/OTKA K 128599). MR and ÁS were supported by the 2017-1.2.1-NKP-2017-00002 Research Grant (Hungarian Brain Research Program) and by the NKFI (National Research, Development and Innovation Office, Hungary) K124098 Research Grant. ÁS was supported by the ÚNKP-20-4-II-BME-13 New National Excellence Program of the Ministry for Innovation and Technology from the source of the National Research, Development, and Innovation Fund. The funding sources had no role in study design; in the collection, analysis and interpretation of data; in the writing of the report; and in the decision to submit the article for publication. 
RUNNING HEAD: Schizotypy \& pattern separation/false memory

\section{Competing interests}

The authors have no competing interests to declare that are relevant to the content of this article.

\section{Ethics approval}

All procedures performed in studies involving human participants were in accordance with the ethical standards of the institutional and/or national research committee and with the 1964 Helsinki Declaration and its later amendments or comparable ethical standards. The study was approved by the United Ethical Review Committee for Research in Psychology, Hungary (2016/032).

\section{Consent to participate}

Informed consent was obtained from all individual participants included in the study.

\section{References}

1. Ettinger U, Meyhöfer I, Steffens M, et al (2014) Genetics, Cognition, and Neurobiology of Schizotypal Personality: A Review of the Overlap with Schizophrenia. Front Psychiatry 5:. https://doi.org/10.3389/fpsyt.2014.00018

2. Ettinger U, Mohr C, Gooding DC, et al (2015) Cognition and Brain Function in Schizotypy: A Selective Review. Schizophr Bull 41:S417-S426. https://doi.org/10.1093/schbul/sbu190

3. Barrantes-Vidal N, Grant P, Kwapil TR (2015) The Role of Schizotypy in the Study of the Etiology of Schizophrenia Spectrum Disorders. Schizophr Bull 41:S408-S416. https://doi.org/10.1093/schbul/sbu191

4. Simeone JC, Ward AJ, Rotella P, et al (2015) An evaluation of variation in published estimates of schizophrenia prevalence from 1990-2013: a systematic literature review. BMC Psychiatry 15:193. https://doi.org/10.1186/s12888-015-0578-7

5. Claridge G (1997) Schizotypy: Implications for illness and health. Oxford University Press, New York, NY, US

6. Zierhut K, Bogerts B, Schott B, et al (2010) The role of hippocampus dysfunction in deficient memory encoding and positive symptoms in schizophrenia. Psychiatry Research: Neuroimaging 183:187-194. https://doi.org/10.1016/j.pscychresns.2010.03.007

7. Tamminga CA, Stan AD, Wagner AD (2010) The hippocampal formation in schizophrenia. American Journal of Psychiatry 167:1178-1193

8. Tamminga CA, Southcott S, Sacco C, et al (2012) Glutamate Dysfunction in Hippocampus: Relevance of Dentate Gyrus and CA3 Signaling. Schizophrenia Bulletin 38:927-935. https://doi.org/10.1093/schbul/sbs062

9. Tulving E, Markowitsch HJ (1998) Episodic and declarative memory: Role of the hippocampus. Hippocampus 8:198-204. https://doi.org/10.1002/(SICI)1098-1063(1998)8:3<198::AIDHIPO2>3.0.CO;2-G

10. Keresztes A, Ngo CT, Lindenberger U, et al (2018) Hippocampal Maturation Drives Memory from Generalization to Specificity. Trends in Cognitive Sciences 22:676-686. https://doi.org/10.1016/j.tics.2018.05.004

11. Ngo CT, Michelmann S, Olson IR, Newcombe NS (2021) Pattern separation and pattern completion: Behaviorally separable processes? Mem Cogn 49:193-205. 
https://doi.org/10.3758/s13421-020-01072-y

12. Yassa MA, Stark CEL (2011) Pattern separation in the hippocampus. Trends in Neurosciences 34:515-525. https://doi.org/10.1016/j.tins.2011.06.006

13. Das T, Ivleva EI, Wagner AD, et al (2014) Loss of pattern separation performance in schizophrenia suggests dentate gyrus dysfunction. Schizophrenia Research 159:193-197. https://doi.org/10.1016/j.schres.2014.05.006

14. Martinelli C, Shergill SS (2015) Clarifying the role of pattern separation in schizophrenia: The role of recognition and visual discrimination deficits. Schizophrenia Research 166:328-333. https://doi.org/10.1016/j.schres.2015.06.004

15. Kraguljac NV, Carle M, Frölich MA, et al (2018) Mnemonic Discrimination Deficits in FirstEpisode Psychosis and a Ketamine Model Suggests Dentate Gyrus Pathology Linked to NMethyl-D-Aspartate Receptor Hypofunction. Biological Psychiatry: Cognitive Neuroscience and Neuroimaging 3:231-238. https://doi.org/10.1016/j.bpsc.2017.02.005

16. Shohamy D, Mihalakos P, Chin R, et al (2010) Learning and Generalization in Schizophrenia: Effects of Disease and Antipsychotic Drug Treatment. Biological Psychiatry 67:926-932. https://doi.org/10.1016/j.biopsych.2009.10.025

17. Dickinson D, Iannone VN, Wilk CM, Gold JM (2004) General and specific cognitive deficits in schizophrenia. Biological Psychiatry 55:826-833. https://doi.org/10.1016/j.biopsych.2003.12.010

18. Bleuler E (1950) Dementia praecox or the group of schizophrenias. International Universities Press, New York, NY

19. Sass LA, Parnas J (2003) Schizophrenia, Consciousness, and the Self. Schizophr Bull 29:427444

20. Uhlhaas PJ, Mishara AL (2007) Perceptual Anomalies in Schizophrenia: Integrating Phenomenology and Cognitive Neuroscience. Schizophrenia Bulletin 33:142-156. https://doi.org/10.1093/schbul/sbl047

21. Ferman TJ, Primeau M, Delis D, Jampala CV (1999) Global-local processing in schizophrenia: Hemispheric asymmetry and symptom-specific interference. Journal of the International Neuropsychological Society 5:442-451

22. Johnson SC, Lowery N, Kohler C, Turetsky BI (2005) Global-Local Visual Processing in Schizophrenia: Evidence for an Early Visual Processing Deficit. Biological Psychiatry 58:937946. https://doi.org/10.1016/j.biopsych.2005.04.053

23. Kim M-S, Namgoong Y, Youn T (2008) Effect of organizational strategy on visual memory in patients with schizophrenia. Psychiatry and Clinical Neurosciences 62:427-434. https://doi.org/10.1111/j.1440-1819.2008.01821.x

24. Seidman LJ, Lanca M, Kremen WS, et al (2003) Organizational and Visual Memory Deficits in Schizophrenia and Bipolar Psychoses Using the Rey-Osterrieth Complex Figure: Effects of Duration of Illness. Journal of Clinical and Experimental Neuropsychology 25:949-964. https://doi.org/10.1076/jcen.25.7.949.16482

25. Stark SM, Kirwan CB, Stark CEL (2019) Mnemonic Similarity Task: A Tool for Assessing Hippocampal Integrity. Trends in Cognitive Sciences 23:938-951. https://doi.org/10.1016/j.tics.2019.08.003

26. Polner B, Simor P, Kéri S (2018) Insomnia and intellect mask the positive link between schizotypal traits and creativity. PeerJ 6:e5615. https://doi.org/10.7717/peerj.5615

27. Simor P, Báthori N, Nagy T, Polner B (2019) Poor sleep quality predicts psychotic-like symptoms: an experience sampling study in young adults with schizotypal traits. Acta Psychiatrica Scandinavica 140:135-146. https://doi.org/10.1111/acps.13064

28. Mason OJ, Linney Y, Claridge G (2005) Short scales for measuring schizotypy. Schizophrenia Research 78:293-296. https://doi.org/10.1016/j.schres.2005.06.020

29. Kéri S (2011) Solitary minds and social capital: Latent inhibition, general intellectual functions and social network size predict creative achievements. Psychology of Aesthetics, Creativity, and the Arts 5:215-221. https://doi.org/10.1037/a0022000

30. Koffel E, Watson D (2009) Unusual sleep experiences, dissociation, and schizotypy: Evidence for a common domain. Clinical Psychology Review 29:548-559. https://doi.org/10.1016/j.cpr.2009.06.004 
31. Soldatos CR, Dikeos DG, Paparrigopoulos TJ (2000) Athens Insomnia Scale: validation of an instrument based on ICD-10 criteria. Journal of Psychosomatic Research 48:555-560. https://doi.org/10.1016/S0022-3999(00)00095-7

32. Novak M (2004) Sleep disorders and quality of life. Doctoral dissertation. PhD Diss, Semmelweis Egyetem

33. Cristóbal-Narváez P, Sheinbaum T, Myin-Germeys I, et al (2017) The role of stress-regulation genes in moderating the association of stress and daily-life psychotic experiences. Acta Psychiatrica Scandinavica 136:389-399. https://doi.org/10.1111/acps.12789

34. Spielberger CD, Gorsuch RL, Lushene R, et al (1983) Manual for the State-Trait Anxiety Inventory. Consulting Psychologists Press, Palo Alto, CA

35. Sipos K, Sipos M, Spielberger CD (1988) A State-Trait Anxiety Inventory (STAI) magyar változata. In: Mérei F, Szakács F (eds) Pszichodiagnosztikai Vademecum I/2. Tankönyvkiadó, Budapest, pp 123-136

36. Goldberg DP, Gater R, Sartorius N, et al (1997) The validity of two versions of the GHQ in the WHO study of mental illness in general health care. Psychological Medicine 27:191-197

37. Balajti I, Vokó Z, Ádány R, Kósa K (2007) Validation of the Hungarian versions of the abbreviated sense of coherence (SOC) scale and the general health questionnaire (GHQ-12). Mentálhigiéné és Pszichoszomatika 8:147-161. https://doi.org/10.1556/Mental.8.2007.2.4

38. Stark SM, Yassa MA, Lacy JW, Stark CEL (2013) A task to assess behavioral pattern separation (BPS) in humans: Data from healthy aging and mild cognitive impairment. Neuropsychologia 51:2442-2449. https://doi.org/10.1016/j.neuropsychologia.2012.12.014

39. Lysaker PH, Minor KS, Lysaker JT, et al (2020) Metacognitive function and fragmentation in schizophrenia: Relationship to cognition, self-experience and developing treatments. Schizophrenia Research: Cognition 19:100142. https://doi.org/10.1016/j.scog.2019.100142

40. Kéri S, Nagy O, Kelemen O, et al (2005) Dissociation between medial temporal lobe and basal ganglia memory systems in schizophrenia. Schizophrenia Research 77:321-328. https://doi.org/10.1016/j.schres.2005.03.024

41. Conway MA, Singer JA, Tagini A (2004) The Self and Autobiographical Memory: Correspondence and Coherence. Social Cognition 22:491-529. https://doi.org/10.1521/soco.22.5.491.50768

42. Conway MA, Pleydell-Pearce CW (2000) The construction of autobiographical memories in the self-memory system. Psychological Review 107:261-288. https://doi.org/10.1037/0033295X.107.2.261

43. Cicero DC, Martin EA, Becker TM, Kerns JG (2016) Decreased Self-Concept Clarity in People with Schizophrenia. J Nerv Ment Dis 204:142-147. https://doi.org/10.1097/NMD.0000000000000442

44. Kállai J, Rózsa S, Hupuczi E, et al (2019) Cognitive fusion and affective isolation: Blurred selfconcept and empathy deficits in schizotypy. Psychiatry Research 271:178-186. https://doi.org/10.1016/j.psychres.2018.11.045

45. Polner B, Hupuczi E, Kéri S, Kállai J (2021) Adaptive and maladaptive features of schizotypy clusters in a community sample. Sci Rep 11:16653. https://doi.org/10.1038/s41598-021-95945-0

46. Andreasen NC (1999) A Unitary Model of Schizophrenia: Bleuler's "Fragmented Phrene" as Schizencephaly. Archives of General Psychiatry 56:781-787. https://doi.org/10.1001/archpsyc.56.9.781

47. Hertzig ME, Walker HA (1975) Symptom formation as an expression of disordered information processing in schizophrenic children. J Autism Dev Disord 5:13-24. https://doi.org/10.1007/BF01537969

48. Ross LA, Saint-Amour D, Leavitt VM, et al (2007) Impaired multisensory processing in schizophrenia: Deficits in the visual enhancement of speech comprehension under noisy environmental conditions. Schizophrenia Research 97:173-183. https://doi.org/10.1016/j.schres.2007.08.008

49. Koutsouleris N, Davatzikos C, Borgwardt S, et al (2014) Accelerated Brain Aging in Schizophrenia and Beyond: A Neuroanatomical Marker of Psychiatric Disorders. Schizophrenia Bulletin 40:1140-1153. https://doi.org/10.1093/schbul/sbt142

50. Schnack HG, van Haren NEM, Nieuwenhuis M, et al (2016) Accelerated Brain Aging in 
RUNNING HEAD: Schizotypy \& pattern separation/false memory

Schizophrenia: A Longitudinal Pattern Recognition Study. AJP 173:607-616.

https://doi.org/10.1176/appi.ajp.2015.15070922

51. Dillon SE, Tsivos D, Knight M, et al (2017) The impact of ageing reveals distinct roles for human dentate gyrus and CA3 in pattern separation and object recognition memory. Sci Rep 7:14069. https://doi.org/10.1038/s41598-017-13853-8

52. Ly M, Murray E, Yassa MA (2013) Perceptual versus conceptual interference and pattern separation of verbal stimuli in young and older adults. Hippocampus 23:425-430. https://doi.org/10.1002/hipo.22110

53. Toner CK, Pirogovsky E, Kirwan CB, Gilbert PE (2009) Visual object pattern separation deficits in nondemented older adults. Learning \& Memory 16:338-342. https://doi.org/10.1101/lm.1315109

54. Weiss A, Heckers S (2001) Neuroimaging of declarative memory in schizophrenia. Scandinavian Journal of Psychology 42:239-250. https://doi.org/10.1111/1467-9450.00234

55. Milev P, Ho B-C, Arndt S, Andreasen NC (2005) Predictive Values of Neurocognition and Negative Symptoms on Functional Outcome in Schizophrenia: A Longitudinal First-Episode Study With 7-Year Follow-Up. AJP 162:495-506. https://doi.org/10.1176/appi.ajp.162.3.495

56. Halverson TF, Orleans-Pobee M, Merritt C, et al (2019) Pathways to functional outcomes in schizophrenia spectrum disorders: Meta-analysis of social cognitive and neurocognitive predictors. Neuroscience \& Biobehavioral Reviews 105:212-219.

https://doi.org/10.1016/j.neubiorev.2019.07.020 